Network Working Group

Request for Comments: 1323

Obsoletes: RFC 1072, RFC 1185
V. Jacobson

LBL

R. Braden

ISI

D. Borman

Cray Research

May 1992

\title{
TCP Extensions for High Performance
}

Status of This Memo

This RFC specifies an IAB standards track protocol for the Internet community, and requests discussion and suggestions for improvements. Please refer to the current edition of the "IAB Official Protocol Standards" for the standardization state and status of this protocol. Distribution of this memo is unlimited.

\section{Abstract}

This memo presents a set of TCP extensions to improve performance over large bandwidth*delay product paths and to provide reliable operation over very high-speed paths. It defines new TCP options for scaled windows and timestamps, which are designed to provide compatible interworking with $\mathrm{TCP}^{\prime} \mathrm{s}$ that do not implement the extensions. The timestamps are used for two distinct mechanisms: RTTM (Round Trip Time Measurement) and PAWS (Protect Against Wrapped Sequences). Selective acknowledgments are not included in this memo.

This memo combines and supersedes RFC-1072 and RFC-1185, adding additional clarification and more detailed specification. Appendix C summarizes the changes from the earlier RFCs.

\section{TABLE OF CONTENTS}

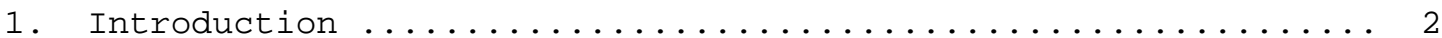

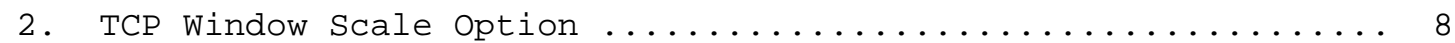

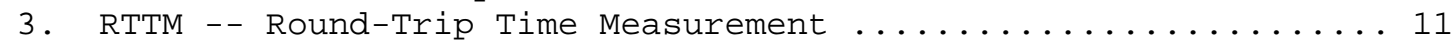

4. PAWS -- Protect Against Wrapped Sequence Numbers .......... 17

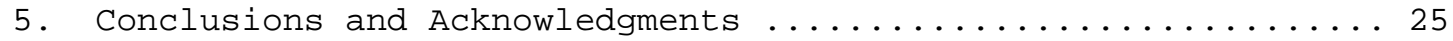

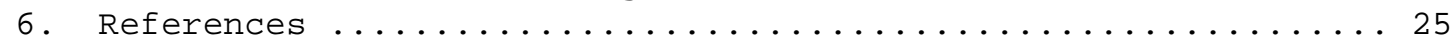

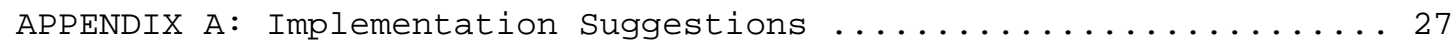

APPENDIX B: Duplicates from Earlier Connection Incarnations ..... 27

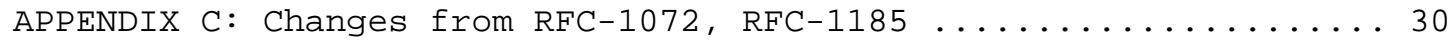

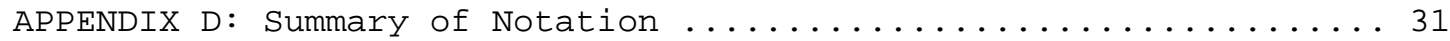

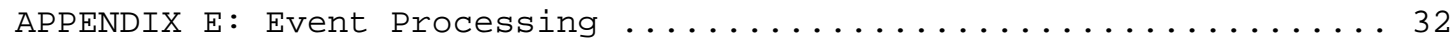

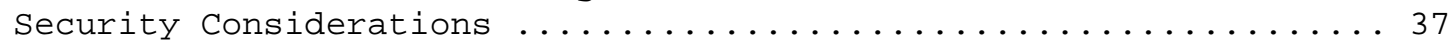




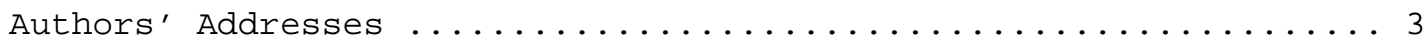

\section{INTRODUCTION}

The TCP protocol [Postel81] was designed to operate reliably over almost any transmission medium regardless of transmission rate, delay, corruption, duplication, or reordering of segments. Production TCP implementations currently adapt to transfer rates in the range of 100 bps to $10 * * 7$ bps and round-trip delays in the range $1 \mathrm{~ms}$ to 100 seconds. Recent work on TCP performance has shown that TCP can work well over a variety of Internet paths, ranging from 800 Mbit/sec I/O channels to $300 \mathrm{bit/sec}$ dial-up modems [Jacobson88a].

The introduction of fiber optics is resulting in ever-higher transmission speeds, and the fastest paths are moving out of the domain for which TCP was originally engineered. This memo defines a set of modest extensions to TCP to extend the domain of its application to match this increasing network capability. It is based upon and obsoletes RFC-1072 [Jacobson88b] and RFC-1185 [Jacobson90b].

There is no one-line answer to the question: "How fast can TCP go?". There are two separate kinds of issues, performance and reliability, and each depends upon different parameters. We discuss each in turn.

\subsection{TCP Performance}

TCP performance depends not upon the transfer rate itself, but rather upon the product of the transfer rate and the round-trip delay. This "bandwidth*delay product" measures the amount of data that would "fill the pipe"; it is the buffer space required at sender and receiver to obtain maximum throughput on the TCP connection over the path, i.e., the amount of unacknowledged data that TCP must handle in order to keep the pipeline full. TCP performance problems arise when the bandwidth*delay product is large. We refer to an Internet path operating in this region as a "long, fat pipe", and a network containing this path as an "LFN" (pronounced "elephan(t)").

High-capacity packet satellite channels (e.g., DARPA's Wideband Net) are LFN's. For example, a DS1-speed satellite channel has a bandwidth*delay product of $10 * * 6$ bits or more; this corresponds to 100 outstanding TCP segments of 1200 bytes each. Terrestrial fiber-optical paths will also fall into the LFN class; for example, a cross-country delay of $30 \mathrm{~ms}$ at a DS3 bandwidth (45Mbs) also exceeds $10 * \star 6$ bits.

There are three fundamental performance problems with the current TCP over LFN paths: 
(1) Window Size Limit

The TCP header uses a 16 bit field to report the receive window size to the sender. Therefore, the largest window that can be used is $2 * \star 16=65 \mathrm{~K}$ bytes.

To circumvent this problem, Section 2 of this memo defines a new TCP option, "Window Scale", to allow windows larger than $2 \star \star 16$. This option defines an implicit scale factor, which is used to multiply the window size value found in a TCP header to obtain the true window size.

(2) Recovery from Losses

Packet losses in an LFN can have a catastrophic effect on throughput. Until recently, properly-operating TCP implementations would cause the data pipeline to drain with every packet loss, and require a slow-start action to recover. Recently, the Fast Retransmit and Fast Recovery algorithms [Jacobson90c] have been introduced. Their combined effect is to recover from one packet loss per window, without draining the pipeline. However, more than one packet loss per window typically results in a retransmission timeout and the resulting pipeline drain and slow start.

Expanding the window size to match the capacity of an LFN results in a corresponding increase of the probability of more than one packet per window being dropped. This could have a devastating effect upon the throughput of TCP over an LFN. In addition, if a congestion control mechanism based upon some form of random dropping were introduced into gateways, randomly spaced packet drops would become common, possible increasing the probability of dropping more than one packet per window.

To generalize the Fast Retransmit/Fast Recovery mechanism to handle multiple packets dropped per window, selective acknowledgments are required. Unlike the normal cumulative acknowledgments of TCP, selective acknowledgments give the sender a complete picture of which segments are queued at the receiver and which have not yet arrived. Some evidence in favor of selective acknowledgments has been published [NBS85], and selective acknowledgments have been included in a number of experimental Internet protocols -- VMTP [Cheriton88], NETBLT [Clark87], and RDP [Velten84], and proposed for OSI TP4 [NBS85]. However, in the non-LFN regime, selective acknowledgments reduce the number of 
packets retransmitted but do not otherwise improve performance, making their complexity of questionable value. However, selective acknowledgments are expected to become much more important in the LFN regime.

RFC-1072 defined a new TCP "SACK" option to send a selective acknowledgment. However, there are important technical issues to be worked out concerning both the format and semantics of the SACK option. Therefore, SACK has been omitted from this package of extensions. It is hoped that SACK can "catch up" during the standardization process.

(3) Round-Trip Measurement

TCP implements reliable data delivery by retransmitting segments that are not acknowledged within some retransmission timeout (RTO) interval. Accurate dynamic determination of an appropriate RTO is essential to TCP performance. RTO is determined by estimating the mean and variance of the measured round-trip time (RTT), i.e., the time interval between sending a segment and receiving an acknowledgment for it [Jacobson88a].

Section 4 introduces a new TCP option, "Timestamps", and then defines a mechanism using this option that allows nearly every segment, including retransmissions, to be timed at negligible computational cost. We use the mnemonic RTTM (Round Trip Time Measurement) for this mechanism, to distinguish it from other uses of the Timestamps option.

\subsection{TCP Reliability}

Now we turn from performance to reliability. High transfer rate enters TCP performance through the bandwidth*delay product. However, high transfer rate alone can threaten TCP reliability by violating the assumptions behind the TCP mechanism for duplicate detection and sequencing.

An especially serious kind of error may result from an accidental reuse of $\mathrm{TCP}$ sequence numbers in data segments. Suppose that an "old duplicate segment", e.g., a duplicate data segment that was delayed in Internet queues, is delivered to the receiver at the wrong moment, so that its sequence numbers falls somewhere within the current window. There would be no checksum failure to warn of the error, and the result could be an undetected corruption of the data. Reception of an old duplicate ACK segment at the transmitter could be only slightly less serious: it is likely to 
lock up the connection so that no further progress can be made, forcing an RST on the connection.

TCP reliability depends upon the existence of a bound on the lifetime of a segment: the "Maximum segment Lifetime" or MSL. An MSL is generally required by any reliable transport protocol, since every sequence number field must be finite, and therefore any sequence number may eventually be reused. In the Internet protocol suite, the MSL bound is enforced by an IP-layer mechanism, the "Time-to-Live" or TTL field.

Duplication of sequence numbers might happen in either of two ways:

(1) Sequence number wrap-around on the current connection

A TCP sequence number contains 32 bits. At a high enough transfer rate, the 32-bit sequence space may be "wrapped" (cycled) within the time that a segment is delayed in queues.

Earlier incarnation of the connection

Suppose that a connection terminates, either by a proper close sequence or due to a host crash, and the same connection (i.e., using the same pair of sockets) is immediately reopened. A delayed segment from the terminated connection could fall within the current window for the new incarnation and be accepted as valid.

Duplicates from earlier incarnations, Case (2), are avoided by enforcing the current fixed MSL of the TCP spec, as explained in Section 5.3 and Appendix B. However, case (1), avoiding the reuse of sequence numbers within the same connection, requires an MSL bound that depends upon the transfer rate, and at high enough rates, a new mechanism is required.

More specifically, if the maximum effective bandwidth at which TCP is able to transmit over a particular path is B bytes per second, then the following constraint must be satisfied for error-free operation:

$$
2 * \star 31 / \mathrm{B}>\mathrm{MSL}(\mathrm{secs})
$$

The following table shows the value for Twrap $=2 * \star 31 / \mathrm{B}$ in seconds, for some important values of the bandwidth $B$ : 


\begin{tabular}{|c|c|c|c|}
\hline Network & $\begin{array}{c}B * 8 \\
\text { bits/sec }\end{array}$ & $\begin{array}{c}\text { B } \\
\text { bytes/sec }\end{array}$ & $\begin{array}{l}\text { Twrap } \\
\text { secs }\end{array}$ \\
\hline ARPANET & $56 \mathrm{kbps}$ & $7 \mathrm{KBps}$ & $3 * 10 * * 5 \quad(\sim 3.6$ days $)$ \\
\hline DS1 & 1.5Mbps & $190 \mathrm{KBps}$ & $10 * * 4 \quad(\sim 3$ hours $)$ \\
\hline Ethernet & $10 \mathrm{Mbps}$ & $1.25 \mathrm{MBps}$ & 1700 ( 30 mins) \\
\hline DS3 & $45 \mathrm{Mbps}$ & $5.6 \mathrm{MBps}$ & 380 \\
\hline FDDI & $100 \mathrm{Mbps}$ & $12.5 \mathrm{MBps}$ & 170 \\
\hline Gigabit & 1 Gbps & $125 \mathrm{MBps}$ & 17 \\
\hline
\end{tabular}

It is clear that wrap-around of the sequence space is not a problem for 56kbps packet switching or even $10 \mathrm{Mbps}$ Ethernets. On the other hand, at DS3 and FDDI speeds, Twrap is comparable to the 2 minute MSL assumed by the TCP specification [Postel81]. Moving towards gigabit speeds, Twrap becomes too small for reliable enforcement by the Internet TTL mechanism.

The 16-bit window field of TCP limits the effective bandwidth $B$ to $2 * \star 16 / \mathrm{RTT}$, where RTT is the round-trip time in seconds

[McKenzie89]. If the RTT is large enough, this limits B to a value that meets the constraint [1] for a large MSL value. For example, consider a transcontinental backbone with an RTT of $60 \mathrm{~ms}$ (set by the laws of physics). With the bandwidth*delay product limited to $64 \mathrm{~KB}$ by the TCP window size, B is then limited to 1.1MBps, no matter how high the theoretical transfer rate of the path. This corresponds to cycling the sequence number space in Twrap $=2000$ secs, which is safe in today's Internet.

It is important to understand that the culprit is not the larger window but rather the high bandwidth. For example, consider a (very large) FDDI LAN with a diameter of $10 \mathrm{~km}$. Using the speed of light, we can compute the RTT across the ring as $(2 * 10 * \star 4) /(3 * 10 * \star 8)=67$ microseconds, and the delay*bandwidth product is then 833 bytes. A TCP connection across this LAN using a window of only 833 bytes will run at the full $100 \mathrm{mbps}$ and can wrap the sequence space in about 3 minutes, very close to the MSL of TCP. Thus, high speed alone can cause a reliability problem with sequence number wrap-around, even without extended windows.

Watson's Delta-T protocol [Watson81] includes network-layer mechanisms for precise enforcement of an MSL. In contrast, the IP 
mechanism for MSL enforcement is loosely defined and even more loosely implemented in the Internet. Therefore, it is unwise to depend upon active enforcement of MSL for TCP connections, and it is unrealistic to imagine setting MSL's smaller than the current values (e.g., 120 seconds specified for TCP).

A possible fix for the problem of cycling the sequence space would be to increase the size of the TCP sequence number field. For example, the sequence number field (and also the acknowledgment field) could be expanded to 64 bits. This could be done either by changing the TCP header or by means of an additional option.

Section 5 presents a different mechanism, which we call PAWS (Protect Against Wrapped Sequence numbers), to extend TCP reliability to transfer rates well beyond the foreseeable upper limit of network bandwidths. PAWS uses the TCP Timestamps option defined in section 4 to protect against old duplicates from the same connection.

\subsection{Using TCP options}

The extensions defined in this memo all use new TCP options. We must address two possible issues concerning the use of TCP options: (1) compatibility and (2) overhead.

We must pay careful attention to compatibility, i.e., to interoperation with existing implementations. The only TCP option defined previously, MSS, may appear only on a SYN segment. Every implementation should (and we expect that most will) ignore unknown options on SYN segments. However, some buggy TCP implementation might be crashed by the first appearance of an option on a non-SYN segment. Therefore, for each of the extensions defined below, TCP options will be sent on non-SYN segments only when an exchange of options on the SYN segments has indicated that both sides understand the extension. Furthermore, an extension option will be sent in a $\langle\mathrm{SYN}, \mathrm{ACK}\rangle$ segment only if the corresponding option was received in the initial <SYN> segment.

A question may be raised about the bandwidth and processing overhead for TCP options. Those options that occur on SYN segments are not likely to cause a performance concern. Opening a TCP connection requires execution of significant special-case code, and the processing of options is unlikely to increase that cost significantly.

On the other hand, a Timestamps option may appear in any data or ACK segment, adding 12 bytes to the 20-byte TCP header. We 
believe that the bandwidth saved by reducing unnecessary retransmissions will more than pay for the extra header bandwidth.

There is also an issue about the processing overhead for parsing the variable byte-aligned format of options, particularly with a RISC-architecture CPU. To meet this concern, Appendix A contains a recommended layout of the options in TCP headers to achieve reasonable data field alignment. In the spirit of Header Prediction, a TCP can quickly test for this layout and if it is verified then use a fast path. Hosts that use this canonical layout will effectively use the options as a set of fixed-format fields appended to the TCP header. However, to retain the philosophical and protocol framework of TCP options, a TCP must be prepared to parse an arbitrary options field, albeit with less efficiency.

Finally, we observe that most of the mechanisms defined in this memo are important for LFN's and/or very high-speed networks. For low-speed networks, it might be a performance optimization to NOT use these mechanisms. A TCP vendor concerned about optimal performance over low-speed paths might consider turning these extensions off for low-speed paths, or allow a user or installation manager to disable them.

\section{TCP WINDOW SCALE OPTION}

\subsection{Introduction}

The window scale extension expands the definition of the TCP window to 32 bits and then uses a scale factor to carry this $32-$ bit value in the 16-bit Window field of the TCP header (SEG.WND in RFC-793). The scale factor is carried in a new TCP option, Window Scale. This option is sent only in a SYN segment (a segment with the SYN bit on), hence the window scale is fixed in each direction when a connection is opened. (Another design choice would be to specify the window scale in every TCP segment. It would be incorrect to send a window scale option only when the scale factor changed, since a TCP option in an acknowledgement segment will not be delivered reliably (unless the ACK happens to be piggy-backed on data in the other direction). Fixing the scale when the connection is opened has the advantage of lower overhead but the disadvantage that the scale factor cannot be changed during the connection.)

The maximum receive window, and therefore the scale factor, is determined by the maximum receive buffer space. In a typical modern implementation, this maximum buffer space is set by default 
but can be overridden by a user program before a TCP connection is opened. This determines the scale factor, and therefore no new user interface is needed for window scaling.

\subsection{Window Scale Option}

The three-byte Window Scale option may be sent in a SYN segment by a TCP. It has two purposes: (1) indicate that the TCP is prepared to do both send and receive window scaling, and (2) communicate a scale factor to be applied to its receive window. Thus, a TCP that is prepared to scale windows should send the option, even if its own scale factor is 1 . The scale factor is limited to a power of two and encoded logarithmically, so it may be implemented by binary shift operations.

TCP Window Scale Option (WSopt):

Kind: 3 Length: 3 bytes

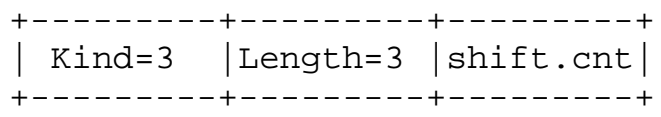

This option is an offer, not a promise; both sides must send Window Scale options in their SYN segments to enable window scaling in either direction. If window scaling is enabled, then the TCP that sent this option will right-shift its true receive-window values by 'shift.cnt' bits for transmission in SEG.WND. The value 'shift.cnt' may be zero (offering to scale, while applying a scale factor of 1 to the receive window).

This option may be sent in an initial <SYN> segment (i.e., a segment with the SYN bit on and the ACK bit off). It may also be sent in a $\langle\mathrm{SYN}, \mathrm{ACK}>$ segment, but only if a Window Scale option was received in the initial <SYN $>$ segment. A Window Scale option in a segment without a SYN bit should be ignored.

The Window field in a SYN (i.e., a <SYN> or <SYN, ACK >) segment itself is never scaled.

\subsection{Using the Window Scale option}

A model implementation of window scaling is as follows, using the notation of RFC-793 [Postel81]:

* All windows are treated as 32-bit quantities for storage in 
the connection control block and for local calculations. This includes the send-window (SND.WND) and the receivewindow (RCV.WND) values, as well as the congestion window.

* The connection state is augmented by two window shift counts, Snd.Wind.Scale and Rcv.Wind.Scale, to be applied to the incoming and outgoing window fields, respectively.

* If a TCP receives a <SYN> segment containing a Window Scale option, it sends its own Window Scale option in the <SYN, ACK> segment.

* The Window Scale option is sent with shift.cnt $=R$, where $R$ is the value that the TCP would like to use for its receive window.

* Upon receiving a SYN segment with a Window Scale option containing shift. cnt $=S$, a TCP sets Snd.Wind.Scale to $S$ and sets Rcv.Wind.Scale to R; otherwise, it sets both Snd.Wind.Scale and Rcv.Wind.Scale to zero.

* The window field (SEG.WND) in the header of every incoming segment, with the exception of SYN segments, is left-shifted by snd.Wind.Scale bits before updating SND.WND:

SND.WND $=$ SEG.WND $<$ Snd.Wind.Scale

(assuming the other conditions of RFC793 are met, and using the "C" notation "<<" for left-shift).

* The window field (SEG.WND) of every outgoing segment, with the exception of SYN segments, is right-shifted by RCV.Wind.Scale bits:

SEG.WND $=$ RCV.WND $>$ RCV.Wind.Scale.

TCP determines if a data segment is "old" or "new" by testing whether its sequence number is within $2 * \star 31$ bytes of the left edge of the window, and if it is not, discarding the data as "old". To insure that new data is never mistakenly considered old and viceversa, the left edge of the sender's window has to be at most $2 \star \star 31$ away from the right edge of the receiver's window. Similarly with the sender's right edge and receiver's left edge. Since the right and left edges of either the sender's or receiver's window differ by the window size, and since the sender and receiver windows can be out of phase by at most the window size, the above constraints imply that 2 * the max window size 
must be less than $2 * * 31$, or

max window $<2 * * 30$

Since the max window is $2 * \star S$ (where $S$ is the scaling shift count) times at most $2 * \star 16-1$ (the maximum unscaled window), the maximum window is guaranteed to be $<2 * 30$ if $S<=14$. Thus, the shift count must be limited to 14 (which allows windows of $2 * \star 30=1$ Gbyte). If a Window Scale option is received with a shift.cnt value exceeding 14, the TCP should log the error but use 14 instead of the specified value.

The scale factor applies only to the Window field as transmitted in the TCP header; each TCP using extended windows will maintain the window values locally as 32-bit numbers. For example, the "congestion window" computed by slow start and Congestion Avoidance is not affected by the scale factor, so window scaling will not introduce quantization into the congestion window.

\section{RTTM: ROUND-TRIP TIME MEASUREMENT}

\subsection{Introduction}

Accurate and current RTT estimates are necessary to adapt to changing traffic conditions and to avoid an instability known as "congestion collapse" [Nagle84] in a busy network. However, accurate measurement of RTT may be difficult both in theory and in implementation.

Many TCP implementations base their RTT measurements upon a sample of only one packet per window. While this yields an adequate approximation to the RTT for small windows, it results in an unacceptably poor RTT estimate for an LFN. If we look at RTT estimation as a signal processing problem (which it is), a data signal at some frequency, the packet rate, is being sampled at a lower frequency, the window rate. This lower sampling frequency violates Nyquist's criteria and may therefore introduce "aliasing" artifacts into the estimated RTT [Hamming77].

A good RTT estimator with a conservative retransmission timeout calculation can tolerate aliasing when the sampling frequency is "close" to the data frequency. For example, with a window of 8 packets, the sample rate is $1 / 8$ the data frequency -- less than an order of magnitude different. However, when the window is tens or hundreds of packets, the RTT estimator may be seriously in error, resulting in spurious retransmissions.

If there are dropped packets, the problem becomes worse. Zhang 
[Zhang86], Jain [Jain86] and Karn [Karn87] have shown that it is not possible to accumulate reliable RTT estimates if retransmitted segments are included in the estimate. Since a full window of data will have been transmitted prior to a retransmission, all of the segments in that window will have to be ACKed before the next RTT sample can be taken. This means at least an additional window's worth of time between RTT measurements and, as the error rate approaches one per window of data (e.g., 10**-6 errors per bit for the Wideband satellite network), it becomes effectively impossible to obtain a valid RTT measurement.

A solution to these problems, which actually simplifies the sender substantially, is as follows: using TCP options, the sender places a timestamp in each data segment, and the receiver reflects these timestamps back in ACK segments. Then a single subtract gives the sender an accurate RTT measurement for every ACK segment (which will correspond to every other data segment, with a sensible receiver). We call this the RTTM (Round-Trip Time Measurement) mechanism.

It is vitally important to use the RTTM mechanism with big windows; otherwise, the door is opened to some dangerous instabilities due to aliasing. Furthermore, the option is probably useful for all TCP's, since it simplifies the sender.

\subsection{TCP Timestamps Option}

TCP is a symmetric protocol, allowing data to be sent at any time in either direction, and therefore timestamp echoing may occur in either direction. For simplicity and symmetry, we specify that timestamps always be sent and echoed in both directions. For efficiency, we combine the timestamp and timestamp reply fields into a single TCP Timestamps Option. 
TCP Timestamps Option (TSopt):

Kind: 8

Length: 10 bytes

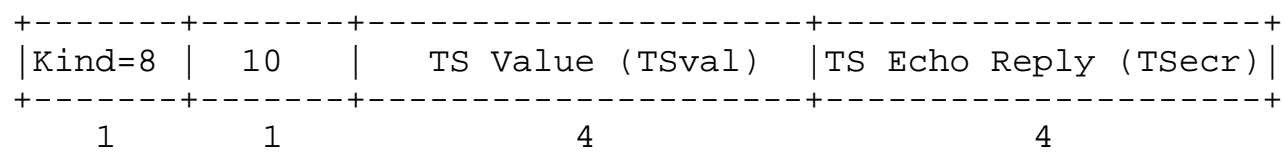

The Timestamps option carries two four-byte timestamp fields. The Timestamp Value field (TSval) contains the current value of the timestamp clock of the TCP sending the option.

The Timestamp Echo Reply field (TSecr) is only valid if the ACK bit is set in the TCP header; if it is valid, it echos a timestamp value that was sent by the remote TCP in the TSval field of a Timestamps option. When TSecr is not valid, its value must be zero. The TSecr value will generally be from the most recent Timestamp option that was received; however, there are exceptions that are explained below.

A TCP may send the Timestamps option (TSopt) in an initial <SYN> segment (i.e., segment containing a SYN bit and no ACK bit), and may send a TSopt in other segments only if it received a TSopt in the initial <SYN> segment for the connection.

\subsection{The RTTM Mechanism}

The timestamp value to be sent in TSval is to be obtained from a (virtual) clock that we call the "timestamp clock". Its values must be at least approximately proportional to real time, in order to measure actual RTT.

The following example illustrates a one-way data flow with segments arriving in sequence without loss. Here A, B, C... represent data blocks occupying successive blocks of sequence numbers, and ACK (A),... represent the corresponding cumulative acknowledgments. The two timestamp fields of the Timestamps option are shown symbolically as <TSval=x, TSecr=y . Each TSecr field contains the value most recently received in a TSval field. 
TCP A

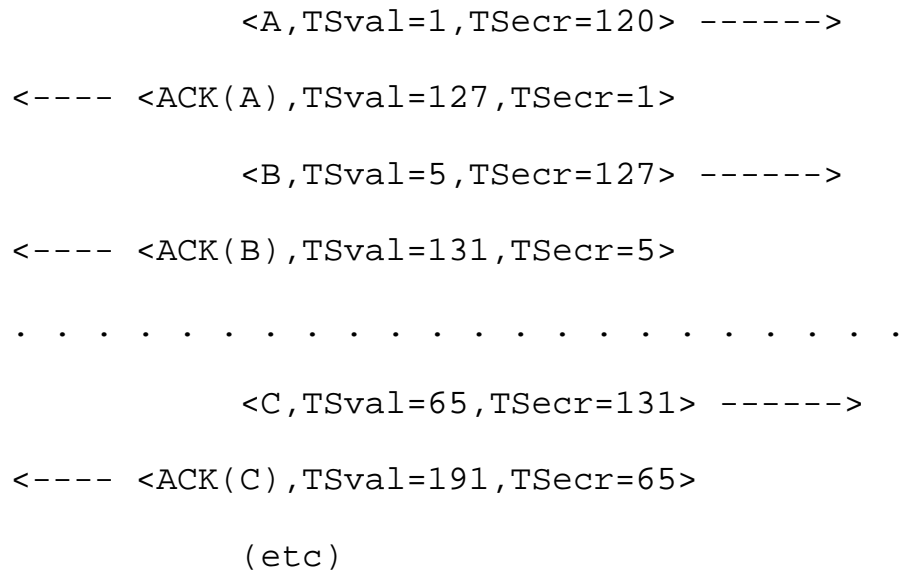

The dotted line marks a pause (60 time units long) in which A had nothing to send. Note that this pause inflates the RTT which B could infer from receiving TSecr=131 in data segment $C$. Thus, in one-way data flows, RTTM in the reverse direction measures a value that is inflated by gaps in sending data. However, the following rule prevents a resulting inflation of the measured RTT:

A TSecr value received in a segment is used to update the averaged RTT measurement only if the segment acknowledges some new data, i.e., only if it advances the left edge of the send window.

Since TCP B is not sending data, the data segment $C$ does not acknowledge any new data when it arrives at B. Thus, the inflated RTTM measurement is not used to update $B^{\prime}$ S RTTM measurement.

\subsection{Which Timestamp to Echo}

If more than one Timestamps option is received before a reply segment is sent, the TCP must choose only one of the TSvals to echo, ignoring the others. To minimize the state kept in the receiver (i.e., the number of unprocessed TSvals), the receiver should be required to retain at most one timestamp in the connection control block. 
There are three situations to consider:

(A) Delayed ACKs.

Many TCP's acknowledge only every Kth segment out of a group of segments arriving within a short time interval; this policy is known generally as "delayed ACKs". The data-sender TCP must measure the effective RTT, including the additional time due to delayed ACKs, or else it will retransmit unnecessarily. Thus, when delayed ACKs are in use, the receiver should reply with the TSval field from the earliest unacknowledged segment.

(B) A hole in the sequence space (segment(s) have been lost).

The sender will continue sending until the window is filled, and the receiver may be generating ACKs as these out-of-order segments arrive (e.g., to aid "fast retransmit").

The lost segment is probably a sign of congestion, and in that situation the sender should be conservative about retransmission. Furthermore, it is better to overestimate than underestimate the RTT. An ACK for an out-of-order segment should therefore contain the timestamp from the most recent segment that advanced the window.

The same situation occurs if segments are re-ordered by the network.

(C) A filled hole in the sequence space.

The segment that fills the hole represents the most recent measurement of the network characteristics. On the other hand, an RTT computed from an earlier segment would probably include the sender's retransmit time-out, badly biasing the sender's average RTT estimate. Thus, the timestamp from the latest segment (which filled the hole) must be echoed.

An algorithm that covers all three cases is described in the following rules for Timestamps option processing on a synchronized connection:

(1) The connection state is augmented with two 32-bit slots: TS.Recent holds a timestamp to be echoed in TSecr whenever a segment is sent, and Last.ACK. sent holds the ACK field from the last segment sent. Last.ACK. sent will equal RCV.NXT except when ACKs have been delayed. 
(2) If Last.ACK. sent falls within the range of sequence numbers of an incoming segment:

SEG.SEQ < = Last.ACK. sent < SEG.SEQ + SEG.LEN

then the TSval from the segment is copied to TS.Recent; otherwise, the TSval is ignored.

(3) When a TSopt is sent, its TSecr field is set to the current TS.Recent value.

The following examples illustrate these rules. Here A, B, C... represent data segments occupying successive blocks of sequence numbers, and ACK $(\mathrm{A}), \ldots$ represent the corresponding acknowledgment segments. Note that $\mathrm{ACK}(\mathrm{A})$ has the same sequence number as B. We show only one direction of timestamp echoing, for clarity.

o Packets arrive in sequence, and some of the ACKs are delayed.

By Case (A), the timestamp from the oldest unacknowledged segment is echoed.

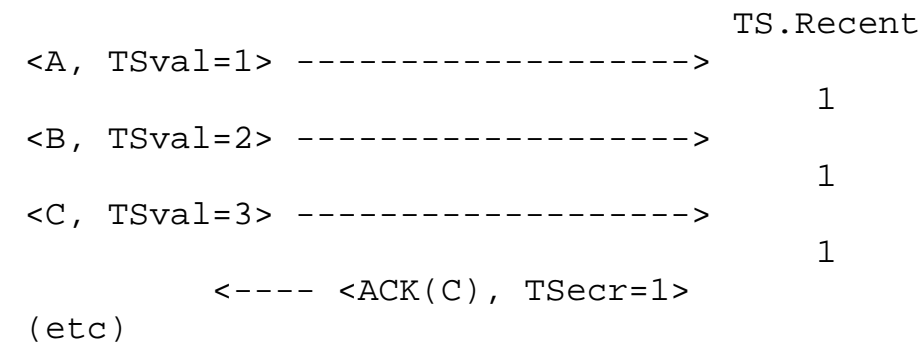

- Packets arrive out of order, and every packet is acknowledged.

By Case (B), the timestamp from the last segment that advanced the left window edge is echoed, until the missing segment arrives; it is echoed according to case (C). The same sequence would occur if segments B and D were lost and retransmitted. . 


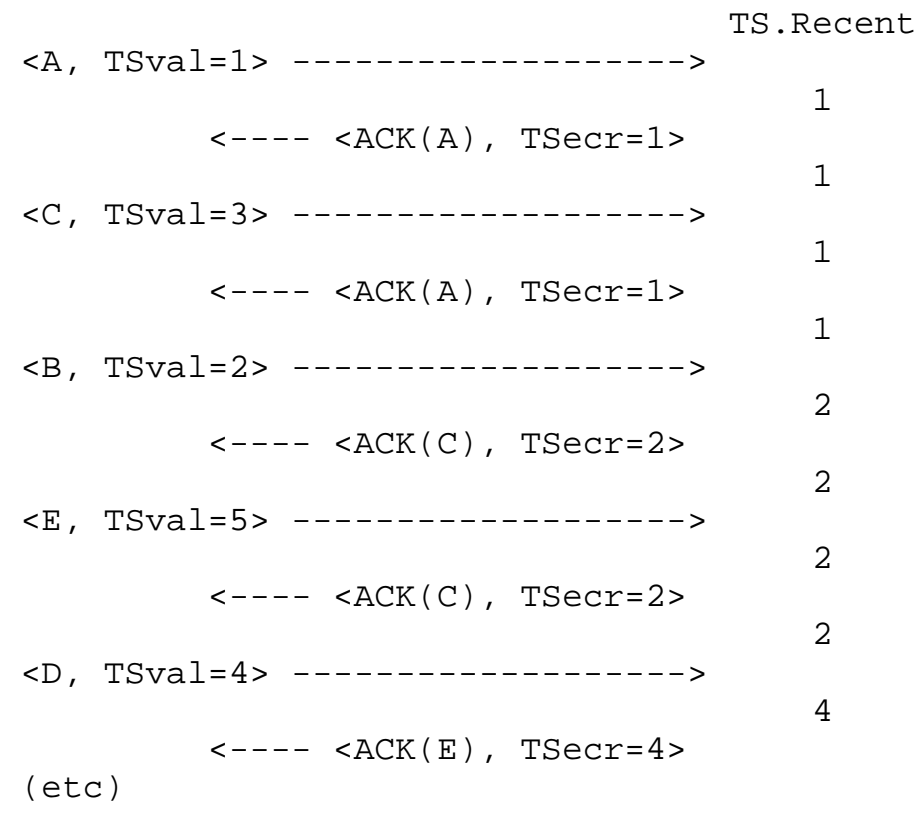

\section{PAWS: PROTECT AGAINST WRAPPED SEQUENCE NUMBERS}

\subsection{Introduction}

Section 4.2 describes a simple mechanism to reject old duplicate segments that might corrupt an open TCP connection; we call this mechanism PAWS (Protect Against Wrapped Sequence numbers). PAWS operates within a single TCP connection, using state that is saved in the connection control block. Section 4.3 and Appendix C discuss the implications of the PAWS mechanism for avoiding old duplicates from previous incarnations of the same connection.

\subsection{The PAWS Mechanism}

PAWS uses the same TCP Timestamps option as the RTTM mechanism described earlier, and assumes that every received TCP segment (including data and ACK segments) contains a timestamp SEG.TSval whose values are monotone non-decreasing in time. The basic idea is that a segment can be discarded as an old duplicate if it is received with a timestamp SEG.TSval less than some timestamp recently received on this connection.

In both the PAWS and the RTTM mechanism, the "timestamps" are 32- 
bit unsigned integers in a modular 32-bit space. Thus, "less than" is defined the same way it is for TCP sequence numbers, and the same implementation techniques apply. If $s$ and $t$ are timestamp values, $s<t$ if $0<(t-s)<2 * * 31$, computed in unsigned 32-bit arithmetic.

The choice of incoming timestamps to be saved for this comparison must guarantee a value that is monotone increasing. For example, we might save the timestamp from the segment that last advanced the left edge of the receive window, i.e., the most recent insequence segment. Instead, we choose the value TS.Recent introduced in section 3.4 for the RTTM mechanism, since using a common value for both PAWS and RTTM simplifies the implementation of both. As Section 3.4 explained, TS.Recent differs from the timestamp from the last in-sequence segment only in the case of delayed ACKs, and therefore by less than one window. Either choice will therefore protect against sequence number wrap-around.

RTTM was specified in a symmetrical manner, so that TSval timestamps are carried in both data and ACK segments and are echoed in TSecr fields carried in returning ACK or data segments. PAWS submits all incoming segments to the same test, and therefore protects against duplicate ACK segments as well as data segments. (An alternative un-symmetric algorithm would protect against old duplicate ACKs: the sender of data would reject incoming ACK segments whose TSecr values were less than the TSecr saved from the last segment whose ACK field advanced the left edge of the send window. This algorithm was deemed to lack economy of mechanism and symmetry.)

TSval timestamps sent on $\{S Y N\}$ and $\{S Y N, A C K\}$ segments are used to initialize PAWS. PAWS protects against old duplicate non-SYN segments, and duplicate SYN segments received while there is a synchronized connection. Duplicate $\{\mathrm{SYN}\}$ and $\{\mathrm{SYN}, \mathrm{ACK}\}$ segments received when there is no connection will be discarded by the normal 3-way handshake and sequence number checks of TCP.

It is recommended that RST segments NOT carry timestamps, and that RST segments be acceptable regardless of their timestamp. Old duplicate RST segments should be exceedingly unlikely, and their cleanup function should take precedence over timestamps.

\subsubsection{Basic PAWS Algorithm}

The PAWS algorithm requires the following processing to be performed on all incoming segments for a synchronized connection: 
R1) If there is a Timestamps option in the arriving segment and SEG.TSval < TS.Recent and if TS.Recent is valid (see later discussion), then treat the arriving segment as not acceptable:

Send an acknowledgement in reply as specified in
RFC-793 page 69 and drop the segment.

Note: it is necessary to send an ACK segment in order to retain $\mathrm{TCP}^{\prime} \mathrm{S}$ mechanisms for detecting and recovering from half-open connections. For example, see Figure 10 of RFC-793.

R2) If the segment is outside the window, reject it (normal TCP processing)

R3) If an arriving segment satisfies: SEG.SEQ <= Last.ACK.sent (see Section 3.4), then record its timestamp in TS.Recent.

R4) If an arriving segment is in-sequence (i.e., at the left window edge), then accept it normally.

R5) Otherwise, treat the segment as a normal in-window, outof-sequence TCP segment (e.g., queue it for later delivery to the user).

Steps R2, R4, and R5 are the normal TCP processing steps specified by RFC-793.

It is important to note that the timestamp is checked only when a segment first arrives at the receiver, regardless of whether it is in-sequence or it must be queued for later delivery. Consider the following example.

Suppose the segment sequence: A.1, B.1, C.1, ..., Z.1 has been sent, where the letter indicates the sequence number and the digit represents the timestamp. Suppose also that segment B.1 has been lost. The timestamp in TS.TStamp is 1 (from A.1), so C.1, ..., Z.1 are considered acceptable and are queued. When $B$ is retransmitted as segment B.2 (using the latest timestamp), it fills the hole and causes all the segments through $\mathrm{Z}$ to be acknowledged and passed to the user. The timestamps of the queued segments are *not* inspected again at this time, since they have already been accepted. When B.2 is accepted, TS.Stamp is set to 2 .

This rule allows reasonable performance under loss. A full 
window of data is in transit at all times, and after a loss a full window less one packet will show up out-of-sequence to be queued at the receiver (e.g., up to $2 * \star 30$ bytes of data); the timestamp option must not result in discarding this data.

In certain unlikely circumstances, the algorithm of rules R1-R4 could lead to discarding some segments unnecessarily, as shown in the following example:

\begin{abstract}
Suppose again that segments: A.1, B.1, C.1, ..., Z.1 have been sent in sequence and that segment B.1 has been lost. Furthermore, suppose delivery of some of C.1, ... Z.1 is delayed until AFTER the retransmission B.2 arrives at the receiver. These delayed segments will be discarded unnecessarily when they do arrive, since their timestamps are now out of date.
\end{abstract}

This case is very unlikely to occur. If the retransmission was triggered by a timeout, some of the segments C.1, .. Z.1 must have been delayed longer than the RTO time. This is presumably an unlikely event, or there would be many spurious timeouts and retransmissions. If $B^{\prime}$ s retransmission was triggered by the "fast retransmit" algorithm, i.e., by duplicate ACKs, then the queued segments that caused these ACKs must have been received already .

Even if a segment were delayed past the RTO, the Fast Retransmit mechanism [Jacobson90c] will cause the delayed packets to be retransmitted at the same time as B.2, avoiding an extra RTT and therefore causing a very small performance penalty.

We know of no case with a significant probability of occurrence in which timestamps will cause performance degradation by unnecessarily discarding segments.

\title{
4.2.2 Timestamp Clock
}

It is important to understand that the PAWS algorithm does not require clock synchronization between sender and receiver. The sender's timestamp clock is used to stamp the segments, and the sender uses the echoed timestamp to measure RTT's. However, the receiver treats the timestamp as simply a monotoneincreasing serial number, without any necessary connection to its clock. From the receiver's viewpoint, the timestamp is acting as a logical extension of the high-order bits of the sequence number. 
The receiver algorithm does place some requirements on the frequency of the timestamp clock.

(a) The timestamp clock must not be "too slow".

It must tick at least once for each $2 * * 31$ bytes sent. In fact, in order to be useful to the sender for round trip timing, the clock should tick at least once per window's worth of data, and even with the RFC-1072 window extension, $2 * \star 31$ bytes must be at least two windows.

To make this more quantitative, any clock faster than 1 tick/sec will reject old duplicate segments for link speeds of 8 Gbps. A 1ms timestamp clock will work at link speeds up to 8 Tbps $(8 * 10 * \star 12)$ bps!

(b) The timestamp clock must not be "too fast".

Its recycling time must be greater than MSL seconds. Since the clock (timestamp) is 32 bits and the worst-case MSL is 255 seconds, the maximum acceptable clock frequency is one tick every 59 ns.

However, it is desirable to establish a much longer recycle period, in order to handle outdated timestamps on idle connections (see section 4.2.3), and to relax the MSL requirement for preventing sequence number wrap-around. With a $1 \mathrm{~ms}$ timestamp clock, the 32-bit timestamp will wrap its sign bit in 24.8 days. Thus, it will reject old duplicates on the same connection if MSL is 24.8 days or less. This appears to be a very safe figure; an MSL of 24.8 days or longer can probably be assumed by the gateway system without requiring precise MSL enforcement by the TTL value in the IP layer.

Based upon these considerations, we choose a timestamp clock frequency in the range $1 \mathrm{~ms}$ to 1 sec per tick. This range also matches the requirements of the RTTM mechanism, which does not need much more resolution than the granularity of the retransmit timer, e.g., tens or hundreds of milliseconds.

The PAWS mechanism also puts a strong monotonicity requirement on the sender's timestamp clock. The method of implementation of the timestamp clock to meet this requirement depends upon the system hardware and software.

* Some hosts have a hardware clock that is guaranteed to be monotonic between hardware resets. 
* A clock interrupt may be used to simply increment a binary integer by 1 periodically.

* The timestamp clock may be derived from a system clock that is subject to being abruptly changed, by adding a variable offset value. This offset is initialized to zero. When a new timestamp clock value is needed, the offset can be adjusted as necessary to make the new value equal to or larger than the previous value (which was saved for this purpose).

\subsubsection{Outdated Timestamps}

If a connection remains idle long enough for the timestamp clock of the other TCP to wrap its sign bit, then the value saved in TS.Recent will become too old; as a result, the PAWS mechanism will cause all subsequent segments to be rejected, freezing the connection (until the timestamp clock wraps its sign bit again).

With the chosen range of timestamp clock frequencies (1 sec to $1 \mathrm{~ms}$ ), the time to wrap the sign bit will be between 24.8 days and 24800 days. A TCP connection that is idle for more than 24 days and then comes to life is exceedingly unusual. However, it is undesirable in principle to place any limitation on TCP connection lifetimes.

We therefore require that an implementation of PAWS include a mechanism to "invalidate" the TS.Recent value when a connection is idle for more than 24 days. (An alternative solution to the problem of outdated timestamps would be to send keepalive segments at a very low rate, but still more often than the wrap-around time for timestamps, e.g., once a day. This would impose negligible overhead. However, the TCP specification has never included keepalives, so the solution based upon invalidation was chosen.)

Note that a TCP does not know the frequency, and therefore, the wraparound time, of the other TCP, so it must assume the worst. The validity of TS.Recent needs to be checked only if the basic PAWS timestamp check fails, i.e., only if SEG.TSval < TS.Recent. If TS.Recent is found to be invalid, then the segment is accepted, regardless of the failure of the timestamp check, and rule R3 updates TS.Recent with the TSval from the new segment.

To detect how long the connection has been idle, the TCP may 
update a clock or timestamp value associated with the connection whenever TS.Recent is updated, for example. The details will be implementation-dependent.

\subsubsection{Header Prediction}

"Header prediction" [Jacobson90a] is a high-performance transport protocol implementation technique that is most important for high-speed links. This technique optimizes the code for the most common case, receiving a segment correctly and in order. Using header prediction, the receiver asks the question, "Is this segment the next in sequence?" This question can be answered in fewer machine instructions than the question, "Is this segment within the window?"

Adding header prediction to our timestamp procedure leads to the following recommended sequence for processing an arriving TCP segment:

H1) Check timestamp (same as step R1 above)

H2) Do header prediction: if segment is next in sequence and if there are no special conditions requiring additional processing, accept the segment, record its timestamp, and skip H3.

H3) Process the segment normally, as specified in RFC-793. This includes dropping segments that are outside the window and possibly sending acknowledgments, and queueing in-window, out-of-sequence segments.

Another possibility would be to interchange steps $\mathrm{H} 1$ and $\mathrm{H} 2$, i.e., to perform the header prediction step H2 FIRST, and perform $\mathrm{H} 1$ and $\mathrm{H} 3$ only when header prediction fails. This could be a performance improvement, since the timestamp check in step $\mathrm{H} 1$ is very unlikely to fail, and it requires interval arithmetic on a finite field, a relatively expensive operation. To perform this check on every single segment is contrary to the philosophy of header prediction. We believe that this change might reduce CPU time for TCP protocol processing by up to 5-10\% on high-speed networks.

However, putting $\mathrm{H} 2$ first would create a hazard: a segment from $2 \star \star 32$ bytes in the past might arrive at exactly the wrong time and be accepted mistakenly by the header-prediction step. The following reasoning has been introduced [Jacobson90b] to show that the probability of this failure is negligible. 
If all segments are equally likely to show up as old duplicates, then the probability of an old duplicate exactly matching the left window edge is the maximum segment size (MSS) divided by the size of the sequence space. This ratio must be less than $2 * \star-16$, since MSS must be $<2 * \star 16$; for example, it will be $(2 * \star 12) /(2 \star \star 32)=$ $2 * \star-20$ for an FDDI link. However, the older a segment is, the less likely it is to be retained in the Internet, and under any reasonable model of segment lifetime the probability of an old duplicate exactly at the left window edge must be much smaller than $2 \star \star-16$.

The 16 bit TCP checksum also allows a basic unreliability of one part in $2 * \star 16$. A protocol mechanism whose reliability exceeds the reliability of the TCP checksum should be considered "good enough", i.e., it won't contribute significantly to the overall error rate. We therefore believe we can ignore the problem of an old duplicate being accepted by doing header prediction before checking the timestamp.

However, this probabilistic argument is not universally accepted, and the consensus at present is that the performance gain does not justify the hazard in the general case. It is therefore recommended that $\mathrm{H} 2$ follow $\mathrm{H} 1$.

\subsection{Duplicates from Earlier Incarnations of Connection}

The PAWS mechanism protects against errors due to sequence number wrap-around on high-speed connection. Segments from an earlier incarnation of the same connection are also a potential cause of old duplicate errors. In both cases, the TCP mechanisms to prevent such errors depend upon the enforcement of a maximum segment lifetime (MSL) by the Internet (IP) layer (see Appendix of RFC-1185 for a detailed discussion). Unlike the case of sequence space wrap-around, the MSL required to prevent old duplicate errors from earlier incarnations does not depend upon the transfer rate. If the IP layer enforces the recommended 2 minute MSL of TCP, and if the TCP rules are followed, TCP connections will be safe from earlier incarnations, no matter how high the network speed. Thus, the PAWS mechanism is not required for this case.

We may still ask whether the PAWS mechanism can provide additional security against old duplicates from earlier connections, allowing us to relax the enforcement of MSL by the IP layer. Appendix B explores this question, showing that further assumptions and/or mechanisms are required, beyond those of PAWS. This is not part of the current extension. 


\section{CONCLUSIONS AND ACKNOWLEDGMENTS}

This memo presented a set of extensions to TCP to provide efficient operation over large-bandwidth*delay-product paths and reliable operation over very high-speed paths. These extensions are designed to provide compatible interworking with $\mathrm{TCP}^{\prime} \mathrm{s}$ that do not implement the extensions.

These mechanisms are implemented using new TCP options for scaled windows and timestamps. The timestamps are used for two distinct mechanisms: RTTM (Round Trip Time Measurement) and PAWS (Protect Against Wrapped Sequences).

The Window Scale option was originally suggested by Mike St. Johns of USAF/DCA. The present form of the option was suggested by Mike Karels of UC Berkeley in response to a more cumbersome scheme defined by Van Jacobson. Lixia Zhang helped formulate the PAWS mechanism description in RFC-1185.

Finally, much of this work originated as the result of discussions within the End-to-End Task Force on the theoretical limitations of transport protocols in general and TCP in particular. More recently, task force members and other on the end2end-interest list have made valuable contributions by pointing out flaws in the algorithms and the documentation. The authors are grateful for all these contributions.

\section{REFERENCES}

[Clark87] Clark, D., Lambert, M., and L. Zhang, "NETBLT: A Bulk Data Transfer Protocol", RFC 998, MIT, March 1987.

[Garlick77] Garlick, L., R. Rom, and J. Postel, "Issues in Reliable Host-to-Host Protocols", Proc. Second Berkeley Workshop on Distributed Data Management and Computer Networks, May 1977.

[Hamming77] Hamming, R., "Digital Filters", ISBN 0-13-212571-4, Prentice Hall, Englewood Cliffs, N.J., 1977.

[Cheriton88] Cheriton, D., "VMTP: Versatile Message Transaction Protocol", RFC 1045, Stanford University, February 1988.

[Jacobson88a] Jacobson, V., "Congestion Avoidance and Control", SIGCOMM'88, Stanford, CA., August 1988 .

[Jacobson88b] Jacobson, V., and R. Braden, "TCP Extensions for Long-Delay Paths", RFC-1072, LBL and USC/Information Sciences Institute, October 1988 . 
[Jacobson90a] Jacobson, V., "4BSD Header Prediction", ACM Computer Communication Review, April 1990.

[Jacobson90b] Jacobson, V., Braden, R., and Zhang, L., "TCP Extension for High-Speed Paths", RFC-1185, LBL and USC/Information Sciences Institute, October 1990.

[Jacobson90C] Jacobson, V., "Modified TCP congestion avoidance algorithm", Message to end2end-interest mailing list, April 1990.

[Jain86] Jain, R., "Divergence of Timeout Algorithms for Packet Retransmissions", Proc. Fifth Phoenix Conf. on Comp. and Comm., Scottsdale, Arizona, March 1986.

[Karn87] Karn, P. and C. Partridge, "Estimating Round-Trip Times in Reliable Transport Protocols", Proc. SIGCOMM '87, Stowe, VT, August 1987 .

[McKenzie89] McKenzie, A., "A Problem with the TCP Big Window Option", RFC 1110, BBN STC, August 1989.

[Nagle84] Nagle, J., "Congestion Control in IP/TCP Internetworks", RFC 896, FACC, January 1984.

[NBS85] Colella, R., Aronoff, R., and K. Mills, "Performance Improvements for ISO Transport", Ninth Data Comm Symposium, published in ACM SIGCOMM Comp Comm Review, vol. 15, no. 5, September 1985 .

[Postel81] Postel, J., "Transmission Control Protocol - DARPA Internet Program Protocol Specification", RFC 793, DARPA, September 1981 .

[Velten84] Velten, D., Hinden, R., and J. Sax, "Reliable Data Protocol", RFC 908, BBN, July 1984.

[Watson81] Watson, R., "Timer-based Mechanisms in Reliable Transport Protocol Connection Management", Computer Networks, Vol. 5,1981 .

[Zhang86] Zhang, L., "Why TCP Timers Don't Work Well", Proc. SIGCOMM '86, Stowe, Vt., August 1986. 


\section{APPENDIX A: IMPLEMENTATION SUGGESTIONS}

The following layouts are recommended for sending options on non-SYN segments, to achieve maximum feasible alignment of 32-bit and 64-bit machines.

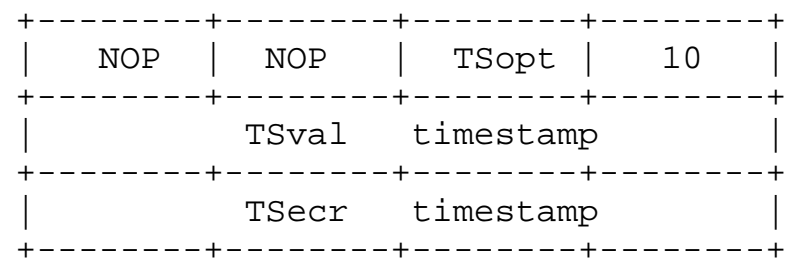

\section{APPENDIX B: DUPLICATES FROM EARLIER CONNECTION INCARNATIONS}

There are two cases to be considered: (1) a system crashing (and losing connection state) and restarting, and (2) the same connection being closed and reopened without a loss of host state. These will be described in the following two sections.

\section{B.1 System Crash with Loss of State}

TCP's quiet time of one MSL upon system startup handles the loss of connection state in a system crash/restart. For an explanation, see for example "When to Keep Quiet" in the TCP protocol specification [Postel81]. The MSL that is required here does not depend upon the transfer speed. The current TCP MSL of 2 minutes seems acceptable as an operational compromise, as many host systems take this long to boot after a crash.

However, the timestamp option may be used to ease the MSL requirements (or to provide additional security against data corruption). If timestamps are being used and if the timestamp clock can be guaranteed to be monotonic over a system crash/restart, i.e., if the first value of the sender's timestamp clock after a crash/restart can be guaranteed to be greater than the last value before the restart, then a quiet time will be unnecessary.

To dispense totally with the quiet time would require that the host clock be synchronized to a time source that is stable over the crash/restart period, with an accuracy of one timestamp clock tick or better. We can back off from this strict requirement to take advantage of approximate clock synchronization. Suppose that the clock is always re-synchronized to within $\mathrm{N}$ timestamp clock 
ticks and that booting (extended with a quiet time, if necessary) takes more than $\mathrm{N}$ ticks. This will guarantee monotonicity of the timestamps, which can then be used to reject old duplicates even without an enforced MSL.

\section{B.2 Closing and Reopening a Connection}

When a TCP connection is closed, a delay of 2 *MSL in TIME-WAIT state ties up the socket pair for 4 minutes (see section 3.5 of [Postel81]. Applications built upon TCP that close one connection and open a new one (e.g., an FTP data transfer connection using Stream mode) must choose a new socket pair each time. The TIMEWAIT delay serves two different purposes:

(a) Implement the full-duplex reliable close handshake of TCP.

The proper time to delay the final close step is not really related to the MSL; it depends instead upon the RTO for the FIN segments and therefore upon the RTT of the path. (It could be argued that the side that is sending a FIN knows what degree of reliability it needs, and therefore it should be able to determine the length of the TIME-WAIT delay for the FIN's recipient. This could be accomplished with an appropriate TCP option in FIN segments.)

Although there is no formal upper-bound on RTT, common network engineering practice makes an RTT greater than 1 minute very unlikely. Thus, the 4 minute delay in TIME-WAIT state works satisfactorily to provide a reliable full-duplex TCP close. Note again that this is independent of MSL enforcement and network speed.

The TIME-WAIT state could cause an indirect performance problem if an application needed to repeatedly close one connection and open another at a very high frequency, since the number of available TCP ports on a host is less than $2 * \star 16$. However, high network speeds are not the major contributor to this problem; the RTT is the limiting factor in how quickly connections can be opened and closed. Therefore, this problem will be no worse at high transfer speeds.

(b) Allow old duplicate segments to expire.

To replace this function of TIME-WAIT state, a mechanism would have to operate across connections. PAWS is defined strictly within a single connection; the last timestamp is TS.Recent is kept in the connection control block, and 


\begin{abstract}
discarded when a connection is closed.
An additional mechanism could be added to the TCP, a per-host cache of the last timestamp received from any connection. This value could then be used in the PAWS mechanism to reject old duplicate segments from earlier incarnations of the connection, if the timestamp clock can be guaranteed to have ticked at least once since the old connection was open. This would require that the TIME-WAIT delay plus the RTT together must be at least one tick of the sender's timestamp clock. Such an extension is not part of the proposal of this RFC.

Note that this is a variant on the mechanism proposed by Garlick, Rom, and Postel [Garlick77], which required each host to maintain connection records containing the highest sequence numbers on every connection. Using timestamps instead, it is only necessary to keep one quantity per remote host, regardless of the number of simultaneous connections to that host.
\end{abstract}


APPENDIX C: CHANGES FROM RFC-1072, RFC-1185

The protocol extensions defined in this document differ in several important ways from those defined in RFC-1072 and RFC-1185.

(a) SACK has been deferred to a later memo.

(b) The detailed rules for sending timestamp replies (see section 3.4) differ in important ways. The earlier rules could result in an under-estimate of the RTT in certain cases (packets dropped or out of order).

(c) The same value TS.Recent is now shared by the two distinct mechanisms RTTM and PAWS. This simplification became possible because of change (b).

(d) An ambiguity in RFC-1185 was resolved in favor of putting timestamps on ACK as well as data segments. This supports the symmetry of the underlying TCP protocol.

(e) The echo and echo reply options of RFC-1072 were combined into a single Timestamps option, to reflect the symmetry and to simplify processing.

(f) The problem of outdated timestamps on long-idle connections, discussed in section 4.2.2, was realized and resolved.

(g) RFC-1185 recommended that header prediction take precedence over the timestamp check. Based upon some scepticism about the probabilistic arguments given in Section 4.2.4, it was decided to recommend that the timestamp check be performed first.

(h) The spec was modified so that the extended options will be sent on $<$ SYN, ACK > segments only when they are received in the corresponding <SYN> segments. This provides the most conservative possible conditions for interoperation with implementations without the extensions.

In addition to these substantive changes, the present RFC attempts to specify the algorithms unambiguously by presenting modifications to the Event Processing rules of RFC-793; see Appendix E. 
APPENDIX D: SUMMARY OF NOTATION

The following notation has been used in this document.

Options

WSopt: $\quad$ TCP Window Scale Option

TSopt: TCP Timestamps Option

Option Fields

shift.cnt: Window scale byte in wSopt.

TSval: 32-bit Timestamp Value field in TSopt.

TSecr: 32-bit Timestamp Reply field in TSopt.

Option Fields in Current Segment

SEG.TSval: TSval field from TSopt in current segment.

SEG.TSecr: TSecr field from TSopt in current segment.

SEG.WSopt: 8-bit value in WSopt

Clock Values

my.TSclock: Local source of 32-bit timestamp values

my.TSclock.rate: Period of my.TSclock (1 ms to 1 sec).

Per-Connection State Variables

TS.Recent: Latest received Timestamp

Last.ACK.sent: Last ACK field sent

Snd.TS.OK: $\quad$ 1-bit flag

Snd.WS.OK: $\quad$-bit flag

Rcv.Wind.Scale: Receive window scale power

Snd.Wind.Scale: Send window scale power 
APPENDIX E: EVENT PROCESSING

Event Processing

OPEN Call

- .

An initial send sequence number (ISS) is selected. Send a SYN segment of the form:

$<\mathrm{SEQ}=\mathrm{ISS}><\mathrm{CTL}=\mathrm{SYN}><\mathrm{TSVal}=\mathrm{my} . \mathrm{TSclock}><$ WSopt $=\mathrm{RCV}$. Wind. Scale $>$

$\cdots$

SEND Call

CLOSED STATE (i.e., TCB does not exist)

$\cdots$

LISTEN STATE

If the foreign socket is specified, then change the connection from passive to active, select an ISS. Send a SYN segment containing the options: <TSval=my.TSclock> and $<$ WSopt $=$ RCV.Wind.Scale $>$. Set SND. UNA to ISS, SND.NXT to ISS+1. Enter SYN-SENT state. ...

SYN-SENT STATE

SYN-RECEIVED STATE

$\cdots$

ESTABLISHED STATE

CLOSE-WAIT STATE

Segmentize the buffer and send it with a piggybacked acknowledgment (acknowledgment value $=$ RCV.NXT) . ...

If the urgent flag is set...

If the Snd.TS.OK flag is set, then include the TCP Timestamps option <TSval=my. TSclock, TSecr=TS. Recent> in each data segment.

Scale the receive window for transmission in the segment header: SEG.WND $=($ SND.WND $>$ RCV.Wind.Scale). 


\section{SEGMENT ARRIVES}

$\cdots$

If the state is LISTEN then

first check for an RST

$\cdots$

second check for an ACK

$\cdots$

third check for a SYN

if the SYN bit is set, check the security. If the ... $\cdots$

If the SEG.PRC is less than the TCB.PRC then continue.

Check for a Window Scale option (WSopt); if one is found, save SEG.WSopt in Snd.Wind.Scale and set Snd.WS.OK flag on. Otherwise, set both Snd.Wind.Scale and Rcv.Wind.Scale to zero and clear snd.WS.OK flag.

Check for a TSopt option; if one is found, save SEG.TSval in the variable TS.Recent and turn on the snd.TS.OK bit.

Set RCV.NXT to SEG.SEQ+1, IRS is set to SEG.SEQ and any other control or text should be queued for processing later. ISS should be selected and a SYN segment sent of the form:

$<\mathrm{SEQ}=\mathrm{ISS}><\mathrm{ACK}=\mathrm{RCV} \cdot \mathrm{NXT}>\langle\mathrm{CTL}=\mathrm{SYN}, \mathrm{ACK}>$

If the snd.WS.OK bit is on, include a WSopt option $<$ WSopt=Rcv.Wind.Scale $>$ in this segment. If the Snd.TS.OK bit is on, include a TSopt <TSval=my.TSclock, TSecr=TS.Recent> in this segment. Last.ACK. sent is set to RCV.NXT.

SND.NXT is set to ISS+1 and SND.UNA to ISS. The connection state should be changed to SYN-RECEIVED. Note that any other incoming control or data (combined with SYN) will be processed in the SYN-RECEIVED state, but processing of SYN and ACK should not be repeated. If the listen was not fully specified (i.e., the foreign socket was not fully specified), then the unspecified fields should be filled in now. 


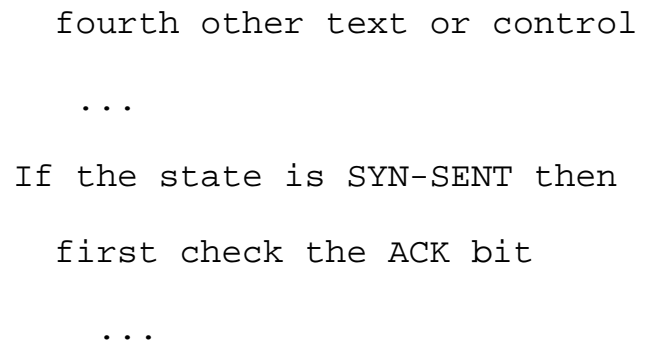


the Snd.WS.OK bit is on, include a WSopt option

$<$ WSopt $=$ RCV.Wind.Scale $>$ in this segment. Last.ACK. sent is set to RCV.NXT .

If there are other controls or text in the segment, queue them for processing after the ESTABLISHED state has been reached, return.

fifth, if neither of the SYN or RST bits is set then drop the segment and return.

Otherwise,

First, check sequence number

SYN-RECEIVED STATE
ESTABLISHED STATE
FIN-WAIT-1 STATE
FIN-WAIT-2 STATE
CLOSE-WAIT STATE
CLOSING STATE
LAST-ACK STATE
TIME-WAIT STATE

Segments are processed in sequence. Initial tests on arrival are used to discard old duplicates, but further processing is done in SEG.SEQ order. If a segment's contents straddle the boundary between old and new, only the new parts should be processed.

Rescale the received window field:

TrueWindow $=$ SEG.WND $\ll$ Snd.Wind.Scale,

and use "TrueWindow" in place of SEG.WND in the following steps.

Check whether the segment contains a Timestamps option and bit snd.TS.OK is on. If so:

If SEG.TSval < TS.Recent, then test whether connection has been idle less than 24 days; if both are true, then the segment is not acceptable; follow steps below for an unacceptable segment.

If SEG.SEQ is equal to Last.ACK.sent, then save SEG.ECopt in variable TS.Recent. 
There are four cases for the acceptability test for an incoming segment :

$$
\cdots
$$

If an incoming segment is not acceptable, an acknowledgment should be sent in reply (unless the RST bit is set, if so drop the segment and return):

$$
<\mathrm{SEQ}=\mathrm{SND} . \mathrm{NXT}><\mathrm{ACK}=\mathrm{RCV} . \mathrm{NXT}><\mathrm{CTL}=\mathrm{ACK}>
$$

Last.ACK.sent is set to SEG.ACK of the acknowledgment. If the Snd.Echo.OK bit is on, include the Timestamps option <TSval=my. TSClock, TSecr=TS. Recent> in this ACK segment. Set Last.ACK. sent to SEG.ACK and send the ACK segment. After sending the acknowledgment, drop the unacceptable segment and return.

$$
\cdots
$$

fifth check the ACK field.

if the ACK bit is off drop the segment and return.

if the ACK bit is on

ESTABLISHED STATE

If SND.UNA $<$ SEG.ACK $=<$ SND.NXT then, set SND. UNA $<-$ SEG.ACK. Also compute a new estimate of round-trip time. If snd.TS.OK bit is on, use my.TSclock - SEG.TSecr; otherwise use the elapsed time since the first segment in the retransmission queue was sent. Any segments on the retransmission queue which are thereby entirely acknowledged...

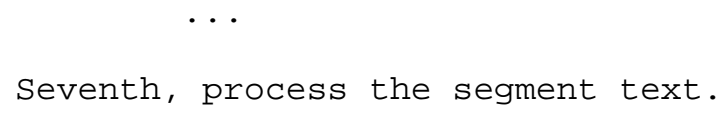




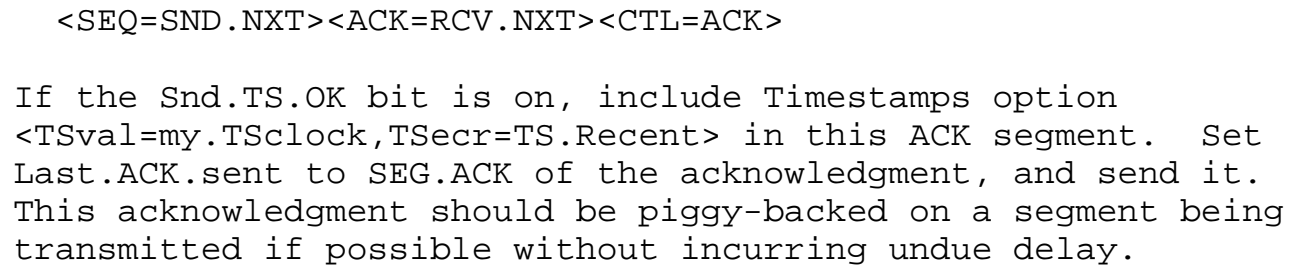

Security Considerations

Security issues are not discussed in this memo.

Authors' Addresses

Van Jacobson

University of California

Lawrence Berkeley Laboratory

Mail stop 46A

Berkeley, CA 94720

Phone: (415) 486-6411

EMail: van@CSAM.LBL.GOV

Bob Braden

University of Southern California

Information Sciences Institute

4676 Admiralty Way

Marina del Rey, CA 90292

Phone: (310) 822-1511

EMail: Braden@ISI.EDU

Dave Borman

Cray Research

655-E Lone Oak Drive

Eagan, MN 55121

Phone: (612) 683-5571

Email: dab@cray.com 